\title{
Bronchoscope Tracking Based on Image Registration Using Multiple Initial Starting Points Estimated by Motion Prediction
}

\author{
Kensaku Mori $^{1}$, Daisuke Deguchi ${ }^{1}$, Takayuki Kitasaka ${ }^{1}$, Yasuhito Suenaga ${ }^{1}$, \\ Hirotsugu Takabatake ${ }^{2}$, Masaki Mori ${ }^{3}$, \\ Hiroshi Natori ${ }^{4}$, and Calvin R. Maurer Jr. ${ }^{5}$ \\ ${ }^{1}$ Graduate School of Information Science, Nagoya University, \\ Furo-cho, Chikusa-ku, Nagoya, Aichi, 464-8603, Japan \\ kensaku@is.nagoya-u.ac.jp \\ 2 Sapporo Minami-Sanjo Hospital \\ ${ }^{3}$ Sapporo Kosei-General Hospital \\ ${ }^{4}$ Keiwakai Nishioka Hospital \\ ${ }^{5}$ Dept. of Neurosurgery, Stanford University
}

\begin{abstract}
This paper presents a method for tracking a bronchoscope based on motion prediction and image registration from multiple initial starting points as a function of a bronchoscope navigation system. We try to improve performance of bronchoscope tracking based on image registration using multiple initial guesses estimated using motion prediction. This method basically tracks a bronchoscopic camera by image registration between real bronchoscopic images and virtual ones derived from CT images taken prior to the bronchoscopic examinations. As an initial guess for image registration, we use multiple starting points to avoid falling into local minima. These initial guesses are computed using the motion prediction results obtained from the Kalman filter's output. We applied the proposed method to nine pairs of X-ray CT images and real bronchoscopic video images. The experimental results showed significant performance in continuous tracking without using any positional sensors.
\end{abstract}

\section{Introduction}

A bronchoscope is a tool for observing the inside of the bronchus or used when doing a biopsy around a suspicious region. A typical video bronchoscope consists of a manipulator that a physician holds and a flexible tube equipped a tiny video camera, a light source, and a working channel. A physician watches a TV monitor that display video images taken by the video camera installed at the tip and proceed to the desired point where biopsy is performed. Due to the progress of CT imaging devices, it is possible to detect lung nodules of a very early stage, especially lung nodules existing on the peripheral areas of the lung. Very- or ultra-thin bronchoscopes are now available and enable biopsies of such lung cancers. However, since the bronchus has a complex tree structure with 
many branches, it is very easy for a physician to get disoriented. Also, physicians have to pay careful attention to important organs such as aorta to avoid severely damaging them. The development of a bronchoscope guidance system that navigates a physician to a target point during bronchoscopic examination is strongly desired.

Although several miniature-size magnetic sensors, which can be installed at the tip of a bronchoscope, are available, the outputs of these sensors are not so precise due to effect of Ferro-magnetic materials existing in examination rooms. There are several reports on overcoming these problems using bronchoscope tracking based on image registration. Even though a very precise magnetic sensor is available, the sensor's output is affected by breathing or heart beat motion. This means that the sensor outputs will shift even when bronchoscopic images does not change. Reference [1] is about trying to guide a bronchoscope using a miniature-size magnetic sensor. However, the navigation accuracy is not very good ( $15 \mathrm{~mm}$ at the right upper lobe). On the other hand, there are some reports on the estimation of bronchoscope motion based on image registration between real bronchoscopic (RB) images and virtual bronchoscopic (VB) images derived from CT images. Bronchoscope tracking methods based on image registration are quite robust against breathing or heart beat motion. Reference [5] estimates bronchoscopic motion by finding the camera parameters of the VB system that gives the most similar VB image to the current RB image. However, it does not work well if the bronchoscope shows large motion between two consecutive frames. Reference [2] proposed an image registration technique that uses bifurcation information of the bronchus. But it is hard to register RB and VB images around positions where no bifurcation structure is observed. Higgins et al. proposed a method for registering $\mathrm{RB}$ and VB images using normalized mutual information [3]. Reference 4] tries to create VB images that are very similar to $\mathrm{RB}$ images by recovering $\mathrm{BRDF}$ from registered $\mathrm{RB}$ and $\mathrm{VB}$ images. Nagao et al. presented a method that predicts bronchoscopic motion using the Kalman filter $[8$. However, the image registration is still trapped in the local maxima.

To solve these problems, this paper proposes a method for bronchoscope tracking based on image registration using multiple initial guesses (starting points). These initial guesses are obtained from bronchoscope motion prediction results calculated with the Kalman filter. Also, we show a method for overlaying anatomical names on RB images using estimated bronchoscopic camera motion.

\section{Method}

\subsection{Overview}

The inputs of the proposed methods are a set of CT images and a bronchoscopic video from the same patient. The output is a sequence of the estimated bronchoscope camera position and the orientation represented in the CT coordinate system (=the VB coordinate system). Bronchoscope tracking is formulated as the search process that finds the best camera position and orientation of the $\mathrm{VB}$ system that generates the most similar VB image to the current RB frame. In 
this search process, multiple starting points are used to avoid falling into local maxima. Figure 1 shows the processing flow.

\subsection{Camera Parameter}

There are two types of camera parameters: (a) an intrinsic camera parameter and (b) an extrinsic camera parameter. The former includes the viewing angle, the offset of the image center from the optical axis, or the distortion parameters. Since these parameters are fixed for each bronchoscopic camera, we obtain these parameters using Tsai's calibration method prior to the bronchoscopic examination. The latter defines the camera position and orientation. Here, we denote the extrinsic camera parameter that generates a VB image $\mathbf{V}$ corresponding to RB image $\mathbf{B}_{k}$ at the frame $k$ as

$$
\mathbf{Q}_{\mathbf{k}}=\left(\begin{array}{cc}
\mathbf{R}_{k} & \mathbf{t}_{k} \\
\mathbf{0}^{T} & 1
\end{array}\right)
$$

where $\mathbf{R}_{k}$ and $\mathbf{t}_{k}$ represent a rotational matrix and a translational vector, respectively. The transformation $\mathbf{Q}_{\mathbf{k}}$ is a transformation matrix from the camera coordinate system to the CT coordinate system. The origin of the camera coordinate system is located at $\mathbf{t}_{k}$ and its orientation is represented by $\mathbf{R}_{k}$ in the CT coordinate system at the $k$-th frame. The extrinsic camera parameter of the camera at the $(k+1)$-th frame is represented as

$$
\mathbf{Q}_{k+1}=\mathbf{Q}_{k} \Delta \mathbf{Q}_{k+1}=\mathbf{Q}_{k}\left(\begin{array}{cc}
\Delta \mathbf{R}_{k+1} & \Delta \mathbf{t}_{k+1} \\
\mathbf{0}^{T} & 1
\end{array}\right),
$$

where $\Delta \mathbf{R}_{k+1}$ and $\Delta \mathbf{t}_{k+1}=\left(\Delta t_{x}, \Delta t_{y}, \Delta t_{z}\right)^{T}$ represent a rotational matrix and translational vector defined in the camera coordinate system at the $k$-th frame and show the change of the camera position and orientation from the $k$-th to $(k+1)$-th frames. For simplicity, we write a matrix $\mathbf{Q}$ composed from a rotational matrix $\mathbf{R}$ and a translational vector $\mathbf{t}$ as $\mathbf{Q}=(\mathbf{R}, \mathbf{t})$. Estimation of the $\mathrm{RB}$ camera motion is equivalent to finding a sequence of the VB camera parameter that generates VB images corresponding to RB images. Therefore, RB camera tracking is done by obtaining a sequence of bronchoscope motion $\Delta \mathbf{Q}_{k+1}=$ $\left(\Delta \mathbf{R}_{k+1}, \Delta \mathbf{t}_{k+1}\right)$.

\subsection{Camera Motion Tracking}

Initial Setup. For the first frames of the RB video, the initial starting point of the search is manually given by comparing the first frame of an $\mathrm{RB}$ video with VB images with changing $\mathbf{R}$ and $\mathbf{t}$.

Motion Prediction. We predict the RB camera position and orientation using the Kalman filter [8]. RB motion is modeled by its velocity and acceleration. We assume that the bronchoscope shows constant acceleration motion in very short period. We input the positions of the bronchoscope at the $1, \cdots, k$-th frames and obtain the predicted position at the $(k+1)$-th frame. 


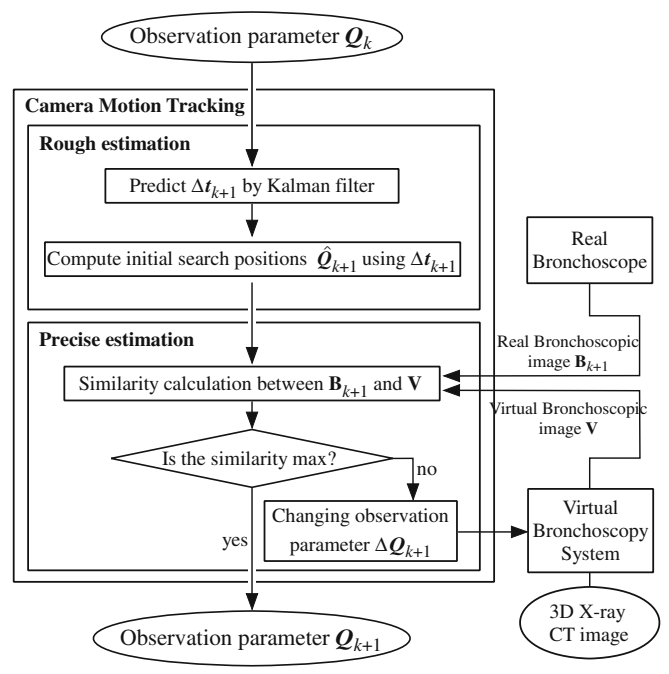

Fig. 1. Processing procedure

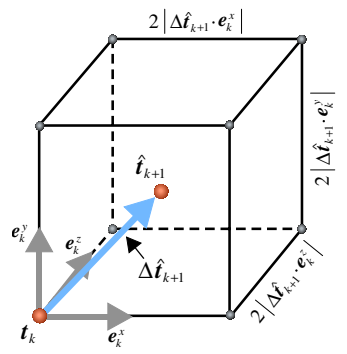

Fig. 2. Allocation of initial points for image registration

Calculation of Multiple Initial Guess for Image Registration. We calculate multiple starting points for image registration using the predicted camera position. First, we obtain the predicted camera position $\hat{\mathbf{t}}_{k+1}$ for the current frame. Then, initial positions for the search of image registration process are computed as the predicted position $\hat{\mathbf{t}}_{k}$ and a set of points $N_{k}$ around $\hat{\mathbf{t}}_{k}$. Here, $N_{k}$ is defined as

$$
N_{k}=\left\{\hat{\mathbf{t}}_{k}+\mathbf{S} v\left|\mathbf{v}=a \mathbf{e}_{k}^{x}+b \mathbf{e}_{k}^{y}+c \mathbf{e}_{k}^{z},\right| a|+| b|+| c \mid=3, a, b, c= \pm 1\right\},
$$

where the vectors $\mathbf{e}_{k}^{x}, \mathbf{e}_{k}^{y}$, and $\mathbf{e}_{k}^{z}$ are the basis vectors that represent the orientation of the VB camera at the $k$-th frame. The matrix $\mathbf{S}$ is calculated as

$$
\mathbf{S}=\left(\begin{array}{ccc}
\left|\Delta \hat{\mathbf{t}}_{k+1} \cdot \mathbf{e}_{k}^{x}\right| & 0 & 0 \\
0 & \left|\Delta \hat{\mathbf{t}}_{k+1} \cdot \mathbf{e}_{k}^{y}\right| & 0 \\
0 & 0 & \left|\Delta \hat{\mathbf{t}}_{k+1} \cdot \mathbf{e}_{k}^{z}\right|
\end{array}\right)
$$

where $\Delta \hat{\mathbf{t}}_{k+1}=\hat{\mathbf{t}}_{k+1}-\mathbf{t}_{k}$ is a vector that represents movement from the camera position $\mathbf{t}_{k}$ at the $k$-th frame to the predicated camera position $\hat{\mathbf{t}}_{k+1}$ at the $(k+1)$-th frame. The initial camera positions defined by Eq. (44) are vertices of a rectangular illustrated in Fig. 2 and $\hat{\mathbf{t}}_{k+1}$.

Multiple Image Registration. We perform image registration between the $\mathrm{RB}$ and VB images by using the initial guesses computed in the previous step. As an initial guess for camera orientation, we use the camera orientation $\mathbf{R}_{k}$ at the the previous frame. So the initial starting point of camera parameter estimation 
in the image registration process is described as $\hat{\mathbf{Q}}_{k+1}=\left(\mathbf{R}_{k}, \mathbf{t}\right)\left(\mathbf{t} \in N_{k}\right)$. We perform the image registration process for each initial starting point by

$$
\Delta \mathbf{Q}_{k+1}^{*}=\arg \max _{\Delta \mathbf{Q}} E\left(\mathbf{B}_{k+1}, \mathbf{V}\left(\hat{\mathbf{Q}}_{k+1} \Delta \mathbf{Q}\right),\right.
$$

where $E(\mathbf{B}, \mathbf{V})$ is the image similarity measure between $\mathbf{B}$ and $\mathbf{V}$. Here, we use the selective image similarity measure presented in 6]. Powell method is used for solving this maximization process. After performing the image registration process for each initial starting point, we select the obtained camera parameter change $\Delta \mathbf{Q}_{k+1}^{*}$ that gives the highest image similarity as the final result of camera motion estimation $\Delta \mathbf{Q}_{k+1}$. The camera parameter at the $(k+1)$-th frame is calculated as $\mathbf{Q}_{k+1}=\hat{\mathbf{Q}}_{k+1} \Delta \mathbf{Q}_{k+1}$. Since more than three frames are required to obtain motion prediction from the Kalman filter, we use $\hat{\mathbf{Q}}_{k+1}=\mathbf{Q}_{k}$ as an initial guess and perform image registration once for each frame if $k<3$. After image registration, we proceed to the next frame.

\subsection{Anatomical Name Display During Bronchoscopy}

It is possible to overlay navigation information on $\mathrm{RB}$ images if the RB camera motion is tracked. Here, we overlay the anatomical names of bronchial branches that are currently observed on RB images. To achieve this function, we extract bronchus regions from CT images. Next, tree structures are extracted from the extracted bronchus regions. Anatomical names are automatically assigned to bronchial branches using the method presented in [7]. Visible branches on RB images are determined using the camera position and orientation of the $\mathrm{RB}$ and tree structure. If visible branches are identified, we overlay their anatomical names on the RB images.

\section{Experiments and Discussion}

We applied the proposed method to nine pairs of CT images and bronchoscopic videos (eight real patients and one phantom). The image acquisition parameters of the CT images are: $512 \times 512$ pixels, $72-341$ slices, $1.25-5.0 \mathrm{~mm}$ slice thickness, and an 0.5-2.0mm reconstruction pitch. $\mathrm{RB}$ videos were taken by BF-240 (Olympus, Tokyo, Japan) and recorded onto digital video tapes. All of the registration task were performed as off-line jobs due to the limitations of computation time. We used a personal computer with dual 3.6GHz Intel Xeon processors and used Linux operating system. We also performed bronchoscope tracking using the previous method (using only one initial starting point and without motion prediction by Kalman filtering) and with multiple initial guesses without motion prediction. In the latter case, the camera position of the previous frame is used as $\hat{\mathbf{t}}_{k}$ ). To process one RB frame, it took 8.70 seconds, while the previous method (using only one initial guess) took 1.01 seconds. We counted the number of frames that were successfully tracked with visual inspection. The results of tracking are shown in Table 1 and bronchoscope tracking are shown in Fig. 3. Also, we tried 
Table 1. Results of bronchoscope motion tracking

\begin{tabular}{|c|c|c|c|c|c|c|}
\hline \multirow[b]{3}{*}{ Case } & \multirow[b]{3}{*}{ Path } & \multirow{3}{*}{$\begin{array}{l}\text { Max movement } \\
\text { in a sec. }[\mathrm{mm}]\end{array}$} & \multirow{3}{*}{$\begin{array}{l}\text { Number } \\
\text { of frames }\end{array}$} & \multicolumn{3}{|c|}{ Num. of successive frames tracked continuously } \\
\hline & & & & \multirow{2}{*}{$\begin{array}{c}\text { Previous } \\
\text { method }\end{array}$} & \multicolumn{2}{|c|}{ Proposed method } \\
\hline & & & & & $\mathrm{w} / \mathrm{o} \mathrm{KF}$ & $\mathrm{w} / \mathrm{KF}$ \\
\hline \multirow{4}{*}{1} & $\mathrm{~A}$ & 35.9 & 993 & 993 & 918 & 993 \\
\hline & $\mathrm{B}$ & 19.5 & 690 & 690 & 690 & 690 \\
\hline & $\mathrm{C}$ & 34.4 & 379 & 82 & 379 & 379 \\
\hline & D & 28.5 & 279 & 279 & 279 & 279 \\
\hline 2 & $\bar{A}$ & 35.0 & 430 & 430 & 430 & 430 \\
\hline \multirow{3}{*}{3} & $\bar{A}$ & 68.4 & 1365 & 278 & 278 & 1365 \\
\hline & B & 43.6 & 965 & 965 & 965 & 965 \\
\hline & $\mathrm{C}$ & 40.6 & 865 & 679 & 859 & 865 \\
\hline 4 & $\mathrm{~A}$ & 26.2 & 300 & 82 & 82 & 82 \\
\hline \multirow{3}{*}{5} & $\overline{\mathrm{A}}$ & 19.1 & 400 & 400 & 400 & 400 \\
\hline & B & 50.6 & 800 & 487 & 477 & 410 \\
\hline & $\mathrm{C}$ & 32.9 & 524 & 353 & 515 & 524 \\
\hline \multirow[b]{2}{*}{6} & $\mathrm{~A}$ & 25.5 & 225 & 140 & 154 & 190 \\
\hline & B & 31.9 & 530 & 457 & 457 & 219 \\
\hline 7 & $\mathrm{~A}$ & 14.2 & 2000 & 339 & 412 & 2000 \\
\hline \multirow[b]{2}{*}{8} & $\overline{\mathrm{A}}$ & 36.9 & 500 & 225 & 354 & 493 \\
\hline & B & 20.5 & 205 & 205 & 205 & 205 \\
\hline \multirow[t]{2}{*}{9} & $\mathrm{~A}$ & 32.8 & 1600 & 467 & 1600 & 1600 \\
\hline & & Total & 13050 & 7551 & 9454 & 12089 \\
\hline
\end{tabular}

to display anatomical names on RB images using the image registration results. Figure 4 shows examples of anatomical display on RB images.

From the results shown in Table 1, we can see that the proposed method showed a greatly improved tracking performance compared to those with the previous method. The total number of frames tracked successfully increased by 4538 frames (roughly equivalent to 150 seconds). The tracking performance was especially much improved in Case $3 \mathrm{~A}$ where the bronchoscope showed large movement between successive frames (see Max. movement in a sec. in Table 1). The previous methods fell into local solution in the search process. However, introduction of the search from multiple initial starting points avoided this local solution and showed good tracking results. Motion prediction for the initial starting points setup also contributed to this improvement. As shown in Table 1. we still failed in tracking (Case 1A or Case 3B). In such cases, bubbles or large bronchi deformation caused by heartbeat were observed on RB images. Both the previous and the proposed methods could not track bronchoscopic motion appropriately. One solution for solving this problem is to pause tracking if such organ deformation or bubbles are detected on RB images and to resume when they disappeared. Since we used multiple initial starting points in the search process, the computation time increased from 1.01 to 8.70 seconds. However, each search process is executed independently. It is quite easy to implement this on cluster-type PCs for reducing computation time. 


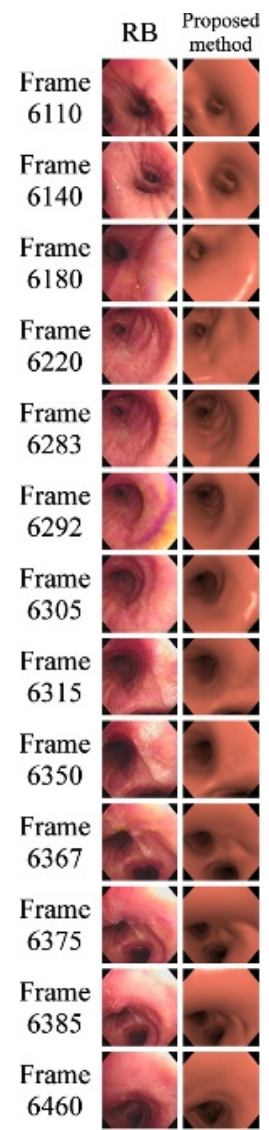

(a) Case $1 \mathrm{C}$

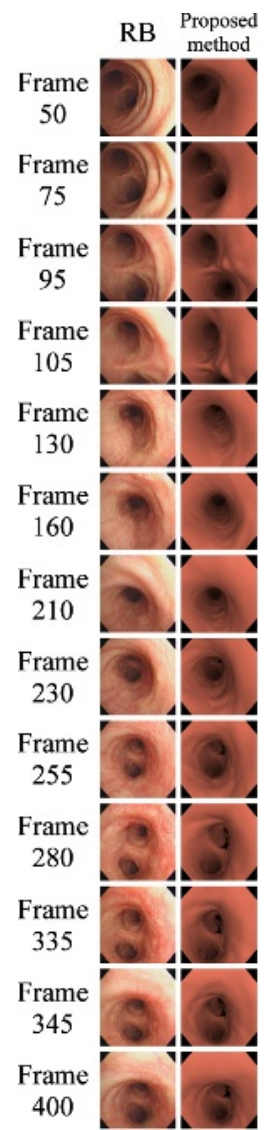

(b) Case $2 \mathrm{~A}$

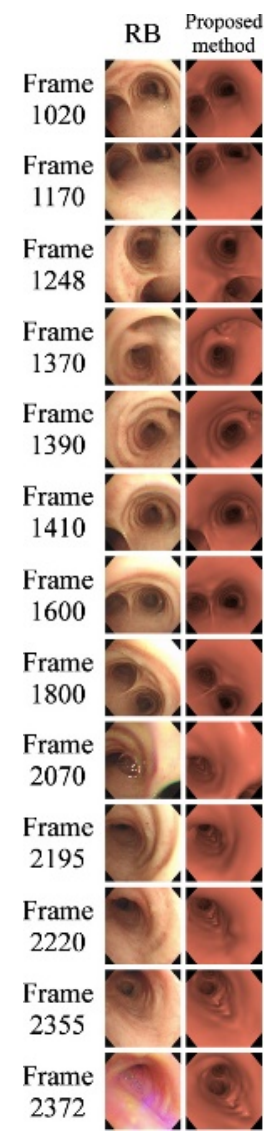

(c) Case $3 \mathrm{~A}$

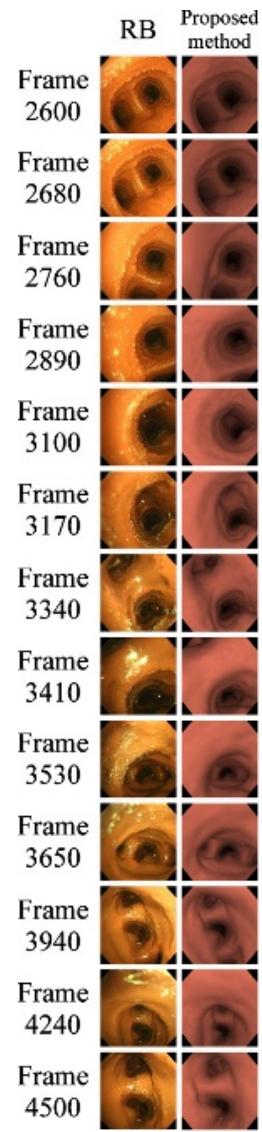

(d) Case $7 \mathrm{~A}$

Fig. 3. Tracking results of the proposed method

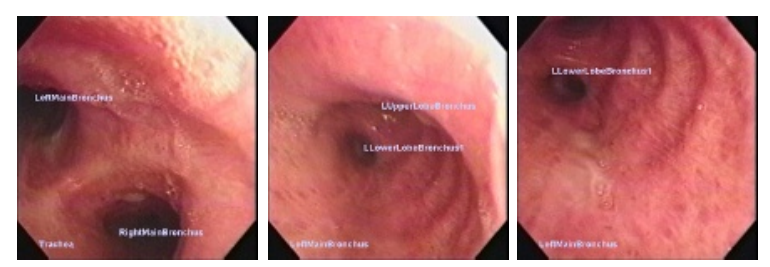

Fig. 4. Anatomical name overlay on RB images using image registration results

\section{Conclusion}

This paper presented a method for bronchoscope tracking that uses utilizes image registration from multiple starting points calculated from motion prediction results. Multiple image registration is performed from the calculated starting 
points to avoid to being trapped in local maxima. The experimental results showed a great improvement in tracking performance. Future work includes: (a) the use of PC clusters to achieve real-time RB camera motion tracking, (b) detection of frames where bubble or large bronchi deformation is observed, (c) further consideration on the allocation of initial stating point for image registration.

Acknowledgements. The authors wish to thank our colleagues for their suggestions and advice. Parts of this research were supported by Grant-In-Aid for Scientific Research from the Ministry of Education, the 21st-Century COE program, a Grant-In-Aid for Scientific Research from the Ministry of Education, Culture, Sports, Science, and Technology, Japan Society for Promotion of Science, a Grant-In-Aid for Cancer Research from the Ministry of Health and Welfare, and Kayamori Foundation of Informational Science Advancement.

\section{References}

1. A. Schneider, H. Hautmann, H. Barfuss, T. Pinkau, F. Peltz, H. Feussner, A. Wichert, "Real-time image tracking of a flexible bronchoscope," Proc. of CARS2004, pp.753-757, 2004

2. I. Bricault, G. Ferretti, and P. Cinquin, "Registration of real and CT-derived virtual bronchoscopic images to assist transbronchial biopsy", IEEE Trans. Med. Imaging, vol. 17, pp. 703-714, 1998.

3. J. P. Helferty, and W. E. Higgins, "Technique for Registering 3D Virtual CT Images to Endoscopic Video," Proceedings of ICIP (International Conference on Image Processing), pp. 893-896, 2001

4. A. J. Chung, F. Deligianni, A. Shah, A. Wells, and G. Z. Yang, "VIS-a-VE: Visual Augmentation for Virtual Environments in Surgical Training," Data Visualization 2005, Eurographics / IEEE VGTC Symposium on Visualization, pp. 101-108, 2005

5. K. Mori, D. Deguchi, J. Sugiyama, Y. Suenaga, J. Toriwaki, C. R. Maurer Jr., H. Takabatake, and H. Natori, "Tracking of a bronchoscope using epipolar geometry analysis and intensity-based image registration of real and virtual endoscopic images," Medical Image Analysis, 6, pp.321-336, 2002

6. D. Deguchi, K. Mori, Y. Suenaga, J. Hasegawa, J. Toriwaki, H. Takabatake, H. Natori, "New Image Similarity Measure for Bronchoscope Tracking Based on Image Registration," Proc. of MICCAI 2003, Lecture Notes in Computer Science,Vol.2878, pp.309-406, 2003

7. K. Mori, S. Ema, T. Kitasaka, Y. Mekada, I. Ide, H. Murase, Y. Suenaga, H. Takabatake, M. Mori, H. Natori, "Automated Nomenclature of Bronchial Branches Extracted from CT Images and Its Application to Biopsy Path Planning in Virtual Bronchoscopy," Proc. of MICCAI 2005, Lecture Notes in Computer Science,Vol.3750, pp.854-861, 2005

8. J. Nagao, K. Mori, T. Enjouji, D. Deguchi, T. Kitasaka, Y. Suenaga, J. Hasegawa, J. Toriwaki, H. Takabatake, H. Natori, "Fast and Accurate Bronchoscope Tracking using Image Registration and Motion Prediction," Proc. of MICCAI 2004, Lecture Notes in Computer Science,Vol.3217, pp.551-558, 2004 